\title{
Shenxian-Shengmai Oral Liquid Reduces Myocardial Oxidative Stress and Protects Myocardium from Ischemia-Reperfusion Injury
}

\author{
Yixiu Zhao Xin Zhang $^{\mathrm{b}}$ Jing Luan ${ }^{\mathrm{b}}$ Buchang Zhao ${ }^{\mathrm{c}} \quad \mathrm{Na} \mathrm{An}^{\mathrm{b}} \quad \mathrm{Na} \mathrm{Sun}^{\mathrm{a}}$ \\ Xinhui Wang ${ }^{\mathrm{a}}$ Tao Zhao ${ }^{c}$ Yanhong Sun ${ }^{\mathrm{b}} \quad$ Zidong Donna Fu ${ }^{\mathrm{a}}$ Yan Zhang ${ }^{\mathrm{a}}$ \\ Baofeng Yang ${ }^{a}$
}

${ }^{a}$ Department of Pharmacology (State-Province Key Laboratories of Biomedicine-Pharmaceutics of China, Key Laboratory of Cardiovascular Medicine Research, Ministry of Education), College of Pharmacy, Harbin Medical University, Harbin, 'Department of Pharmacology, College of Pharmacy, Harbin Medical University, Harbin, 'Xian Buchang Chinese Medicine Cardio Cerebral Disease Hospital, Xian, China

\section{Key Words}

Traditional Chinese medicine $\cdot$ Cardiomyocytes $\cdot$ Antioxidant $\cdot$ Bradyarrhythmia $\cdot$ Oxidative stress

\begin{abstract}
Background/Aims: Shenxian-shengmai (SXSM) oral liquid, a Chinese patent compound medicine, has been used to treat sinus bradyarrhythmias induced by mild sick sinus syndrome in clinical practice. Myocardial ischemia, in particular in serious or right coronary-related heart diseases, can cause bradyarrhythmias and cardiac dysfunction. Moreover, reperfusion of ischemic myocardium is associated with additional myocardial damage known as myocardial ischemia-reperfusion (I/R) injury. This study was designed to evaluate the effects of SXSM on bradyarrhythmias and cardiac dysfunction induced by myocardial I/R injury, and to explore the underlying mechanisms. Methods: Administration of SXSM to adult male Sprague Dawley (SD) rats was achieved orally by gavage and control rats were given equivalent deionized water every day for 14 days. After the last administration, the heart was connected with the Langendorff perfusion apparatus and both groups were subjected to ischemia for 20 min followed by reperfusion for 40 min to induce myocardial I/R injury. Heart rate (HR), left ventricular developed pressure (LVDP), the maximal increase rate of left ventricular pressure $\left(+\mathrm{dp} / \mathrm{dt}_{\max }\right)$ and the maximal decrease rate of left ventricular pressure $\left(-\mathrm{dp} / \mathrm{dt}_{\max }\right)$ were recorded by a physiological signal acquisition system. The heart treated with ischemic preconditioning (IPC) for 3 times at a range of $5 \mathrm{~min} /$ time before ischemia served as a positive control group.


The hearts without I/R injury served as control group. After reperfusion, superoxide dismutase (SOD), glutathione (GSH) and glutathione peroxidase (GSH-Px) activities in the myocardium were determined by appropriate assay kits. Myocardial SOD1 and glutamate cysteine ligase catalytic subunit $(\mathrm{GCLC})$ expression were assessed by western blot analysis. For the in vitro study, SXSM serum was prepared according to the serum pharmacological method and neonatal rat cardiomyocytes were isolated from the heart of new born SD rats. Neonatal rat cardiomyocytes were pretreated with SXSM serum and subjected to $\mathrm{H}_{2} \mathrm{O}_{2}$ or anoxia/ reoxygenation $(A / R)$ treatment to induce oxidative damage. Cell viability was evaluated using a Cell Counting Kit-8 (CCK8) assay. Levels of reactive oxygen species (ROS), SOD, GSH and GSHPx in cardiomyocytes were determined by appropriate assay kits. SOD1 and GCLC expression were assessed by western blot analysis. Buthionine-[S, R]-sulfoximine (BSO), a GCLC inhibitor, and SOD1 siRNA were also used for identifying the cardiac protective targets of SXSM. Results: SXSM and ischemic preconditioning (IPC) significantly increased heart rate during myocardial reperfusion and protected cardiac function against myocardial I/R injury, including an increase in left ventricular diastolic pressure (LVDP), the maximal increase rate of left ventricular pressure $\left(+\mathrm{dp} / \mathrm{dt}_{\text {max }}\right)$ and the maximal decrease rate of left ventricular pressure $\left(-\mathrm{dp} / \mathrm{dt}_{\text {max }}\right)$. We also found that SXSM and IPC improved the expansion of myocardial interstitium, the structural abnormality and morphological changes of cardiomyocytes induced by I/R injury. Meanwhile, SXSM protected cardiomyocytes against the oxidative damage induced by $\mathrm{H}_{2} \mathrm{O}_{2}$ and $A / R$ injury through reducing intracellular ROS levels. Moreover, SXSM increased SOD activity through enhancing SOD1 expression and increased GSH content through promoting GCLC expression as well as GSH-Px activity. BSO and SOD1 siRNA counteracted anti-arrhythmic and cardiac protective effect of SXSM, suggesting that the therapeutic targets of SXSM might be SOD1 and GCLC. Conclusion: SXSM is effective in protecting the myocardium from I/R injury, with myocardial SOD1 and GCLC being the potential therapeutic targets.

\section{Introduction}

C) 2018 The Author(s)

Published by S. Karger AG, Basel

Ischemic heart disease is a major threaten to global health and frequently leads to sudden cardiac death. The most effective therapeutic strategy for ischemic heart disease is perfusion of ischemic myocardium as early as possible in order to improve clinical outcomes of patients. However, reperfusion of ischemic myocardium is often associated with further myocardial damage, which is recognized as myocardial ischemia-reperfusion (I/R) injury $[1,2]$. In addition to inducing myocardial ischemia and hypoxia, myocardial I/R injury alters excitability and conduction system of cardiac muscles, impairs cardiac diastolic and systolic function, and triggers severe myocardial injury and dysfunction, such as cardiac arrhythmias, myocardial energy metabolism failure, myocardial stunning and microvascular dysfunction $[3,4]$. Therefore, protection of the heart from myocardial I/R injury with an aim to improve patient's clinical outcomes is a key research implication in the field of cardiovascular protection.

Pathological mechanisms of myocardial I/R injury are complicated and are yet to be fully understood at present. Extensive studies have shown that oxidative stress induced by oxygen free radicals is an important factor that triggers myocardial cell death and myocardial dysfunction upon reperfusion $[5,6]$. Reactive oxygen species (ROS) are identified as the most straightforward indicator of oxidative stress level and direct cell damage. ROS include a wide array of radicals, such as superoxide anion $\left(\mathrm{O}_{2}^{-}\right)$, hydrogen peroxide $\left(\mathrm{H}_{2} \mathrm{O}_{2}\right)$ and hydroxyl radicals $(\mathrm{OH})$. Under normal circumstances, endogenous antioxidants, such as superoxide dismutase (SOD), catalase (CAT), glutathione (GSH) and glutathione peroxidase (GSH-Px) can effectively scavenge the oxygen free radicals and maintain the homeostasis between generation and degradation of oxygen free radicals. However, when myocardial I/R injury occurs, cardiac intracellular calcium overload can increase single electron reduction in mitochondria, elevate xanthine oxidase synthesis, and enhance catecholamine oxidation and respiratory burst of neutrophil, leading to a rapid increase in ROS production [7-11]. Damage

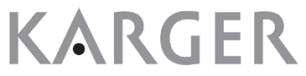


of myocardial cells associated with ROS is achieved through a variety of mechanisms. First, ROS can impair cardiomyocyte membrane to produce lipid peroxides such as malonaldehyde (MDA), which then decreases the membrane fluidity, increases the membrane penetrability and damages cellular structure and function. Second, ROS can react with mitochondrial permeability transition pore and affect the potential of the mitochondrial membrane to disrupt the integrity of the mitochondria and induce cell apoptosis. Third, ROS inhibit the activity of $\mathrm{Ca}^{2+}$-ATPase on the cell membrane, endoplasmic reticulum and mitochondria, resulting in $\mathrm{Ca}^{2+}$ overload and cardiomyocyte apoptosis [12-14]. In addition to an increase in ROS production, I/R injury also increases the consumption of antioxidants such as SOD, GSH, GSH-Px and decreases the cardiac antioxidant capacity, which further aggravates the oxidative stress reaction to induce myocardial damage $[15,16]$.

Shenxian-shengmai (SXSM) oral liquid is a commercially-available Chinese patent medicine and has been used to treat bradyarrhythmias and mild sick sinus syndrome in clinical practice [17-19]. It is formulated in accordance with the traditional Chinese medicine (TCM) theory comprising Panax ginseng C. A. Mey. (Araliaceae), Epimedium brevicornu Maxim (Berberidaceae), Psoralea corylifolia L. (Leguminosae), Lycium barbarum L. (Solanaceae), Ephedra intermedia Schrenk \& C. A. Mey. (Ephedraceae), Asarum sieboldii Miq. (Aristolochiaceae), Salvia miltiorrhiza Bge. (Labiatae) and Whitmania Pigra Whitman (Theromyzon). A previous study has demonstrated that SXSM could accelerate heart rate and improve cardiac function of rats with bradyarrhythmia through increasing cAMP/ cGMP and $\mathrm{Na}^{+}-\mathrm{K}^{+}$-ATPase activity [20]. Besides, SXSM has effective therapeutic effects on sinus bradycardia and can improve the automaticity of sinoatrial nodes in sick sinus rabbits dose-dependently [21-23]. Moreover, SXSM inhibits platelet aggregation and thrombosis induced by laser [24]. However, the possible effects of SXSM on cardiac dysfunction induced by myocardial I/R injury and the underlying mechanisms remain unclear. Consequently, this study was designed to evaluate the effects of SXSM on cardiac dysfunction during myocardial I/R injury, and to explore the potential mechanisms.

\section{Materials and Methods}

\section{Chemicals and reagents}

Dulbecco's Modified Eagle Medium (DMEM) was purchased from Hyclone (Logan, USA). Fetal bovine serum (FBS) was purchased from Gibco (California, USA). Cell Counting Kit-8 (CCK8) assay kit was purchased from Biosharp (Hefei, China). SOD, GSH, GSH-Px assay kits were purchased from Nanjing Jiancheng Bioengineering Institutes (Nanjing, China). ROS detection kit and bicinchoninic acid protein assay reagent kit were purchased from Beyotime Biotechnology (Shanghai, China). BSO was purchased from Aladdin (Shanghai, China). Anti-GCLC antibody was purchased from Abcam (Cambridge, UK). Anti-SOD1 and anti$\beta$-actin antibodies were purchased from Sangon Biotechnology (Shanghai, China). Horseradish peroxidase (HRP)-conjugated, anti-rabbit antibody was purchased from LI-COR Bioscience (Lincoln, USA).

\section{Drug and plant names}

SXSM (Batch No. 150601) used in this study was provided by Buchang Phamaceutical Co., LTD (Heze, China, Patent No. ZL200710019161.5). SXSM comprises eight herbal medicines, including Panax ginseng C. A. Mey. (Araliaceae), Epimedium brevicornu Maxim (Berberidaceae), Psoralea corylifolia L. (Leguminosae), Lycium barbarum L. (Solanaceae), Ephedra intermedia Schrenk \& C. A. Mey. (Ephedraceae), Asarum sieboldii Miq. (Aristolochiaceae), Salvia miltiorrhiza Bge. (Labiatae) and Whitmania Pigra Whitman (Theromyzon). All of the herbal medicines were purchased from Anhui Guangyintang Chinese Medicine Co. Ltd and AnguoKangshenghua Medicine Ltd. (Baoding, China). The preparation method of SXSM was as follows: distillation of Asarum was followed by collection of the volatile oil. Panax ginseng was soaked with water, cut into slices and extracted with ethanol. Ethanol was then recycled and the filtrate was kept in cold temperature. The remaining herbs were decocted with water and filtered. The filtrate was concentrated and mixed with ethanol. The mixture was kept in cold temperature and filtered. The ethanol filtrate was recycled and mixed with the ginseng solution and asarum oil. Acidity of this final solution was adjusted to 


\section{Cellular Physiology Cell Physiol Biochem 2018;48:2503-2516 \begin{tabular}{l|l|l} 
and Biochemistry Published online: 16 August, 2018 & $\begin{array}{l}\text { @ } 2018 \text { The Author(s). Published by S. Karger AG, Basel } \\
\text { www.karger.com/cpb }\end{array}$ \\
\hline
\end{tabular}}

pH 7.3 and the solution was filtered, packaged and sterilized to obtain the finished product of SXSM. The entire production of this herbal preparation met national good manufacturing practice (GMP) standards and the finished product of SXSM liquid complied with the China National Drug Standards WS3-065(Z-010)2003(Z).

\section{Animals}

Adult male Sprague-Dawley SD rats (weighing 200-220 g) were purchased from Liaoning Changsheng Biological Technology Co. (Benxi, China). Animals were fed and housed at temperature of $20-22^{\circ} \mathrm{C}$ and with 40\%-70\% humidity. All animal protocols were approved by the Ethic Committees of Harbin Medical University and were in line with the US National Institutes of Health (NIH) guidelines for the care and use of experimental animals.

\section{Establishment of myocardial I/R injury models}

SD rats were administered SXSM $(3.6 \mathrm{~mL} / \mathrm{kg} /$ day) by gavage once a day for 14 days. After the last medication, the SD rats were anesthetized with sodium pentobarbital $(1.2 \mathrm{mg} / \mathrm{kg})$ and the heart with a section of the aorta was removed from the chest. The heart was then reversely perfused through the aorta with a modified Langendorff perfusion system using oxygenated Krebs-Henseleit (K-H) buffer containing (in mM): $\mathrm{NaCl} 118, \mathrm{NaHCO}_{3} 25$, D-glucose11, $\mathrm{KCl} 4.7, \mathrm{KH}_{2} \mathrm{PO}_{4} 1.2, \mathrm{MgSO}_{4} 1.17$, and $\mathrm{CaCl}_{2} 2.5$; pH 7.4. After perfusion for 10 minutes and cardiac parameters became stable, the heart was subjected to hypoxia by terminating perfusion with the oxygenated K-H buffer. After $20 \mathrm{~min}$, the heart was re-oxygenated by perfusion with oxygenated $\mathrm{K}-\mathrm{H}$ buffer for $40 \mathrm{~min}$. Meanwhile, control rats were given distilled water of the same volume and their hearts were perfused without I/R injury (control group); rats given distilled water with their hearts perfused with I/R injury served as a model group; rats administered with distilled water and the hearts perfused with ischemic preconditioning (IPC) ( 3 sessions at 5 min per session) before ischemia served as a positive control group. Heart rate (HR), left ventricular diastolic pressure (LVDP), the maximal increase rate of left ventricular pressure $\left(+\mathrm{dp} / \mathrm{dt}_{\max }\right)$ and the maximal decrease rate of left ventricular pressure $\left(-\mathrm{dp} / \mathrm{dt}_{\max }\right.$ ) were monitored and recorded using the LabChart flexible data acquisition and analysis software (AD Instruments, California, USA).

\section{Hematoxylin and eosin (HE) staining}

After perfusion, cell fixation was achieved by treating the hearts with $4 \%$ paraformaldehyde, followed by embedding in optimal cutting temperature (OCT) compound, and cut cross-sectionally into 6 - $\mu$ m-thick sections. The structure and morphology of cardiomyocytes and myocardium were observed by Hematoxylin and eosin (H\&E) staining.

\section{Determination of oxidative and reductive parameters of myocardium tissue}

Myocardium tissue obtained from the perfused hearts was used for evaluating myocardial oxidative stress after I/R injury. Antioxidative enzymes including GSH, GSH-Px, SOD were determined by GSH, GSH-Px, SOD assay kits, respectively.

\section{Preparation of SXSM serum}

SD rats were treated with SXSM $(3.6 \mathrm{~mL} / \mathrm{kg} /$ day) or distilled water by gavage twice a day for the first three days. On the fourth day, blood was collected from the abdominal aorta of the rats and left undisturbed for $2 \mathrm{~h}$ at $25^{\circ} \mathrm{C}$. Subsequently, the blood samples were centrifuged at $3500 \mathrm{r} / \mathrm{min}$ for $10 \mathrm{~min}$ to obtain the serum. The serum collected was then centrifuged at $4000 \mathrm{r} / \mathrm{min}$ for $1 \mathrm{~h}$ in a $3 \mathrm{kDa}$ millipore tube to remove macromolecular proteins. The resultant filtrate was filtered again with a $0.22 \mu \mathrm{m}$ millipore filter to obtain serum sample. Then SXSM, serum samples of control and SXSM groups were subjected to high performance liquid chromatography (HPLC) analysis. The analysis was performed with a Cosmosil 5C18-MS-II Packed Column (4.6 ID $\times 250 \mathrm{~mm}$; Tokyo, Japan) maintained at $25{ }^{\circ} \mathrm{C}$ under isocratic flow conditions. The mobile phase used comprised 100\% methanol, 98\% methanol and 95\% methanol at 0-5 min, 5-15 min and 15-20 $\mathrm{min}$, respectively, and the flow rate was set at $1 \mathrm{ml} / \mathrm{min}$. Samples were examined at $254 \mathrm{~nm}$ and identified by an Agilent HPLC-1260 system (California, USA). 


\section{Cellular Physiology Cell Physiol Biochem 2018;48:2503-2516 \begin{tabular}{l|l|l} 
and Biochemistry Published online: 16 August, 2018 & $\begin{array}{l}\text { C) 2018 The Author(s). Published by S. Karger AG, Basel } \\
\text { www.karger.com/cpb }\end{array}$ \\
\hline
\end{tabular}}

\section{Culture of neonatal rat cardiomyocytes}

After sterilizing the skin with 75\% ethanol, the hearts of new-born SD rats were removed from the chest and cut into small pieces in FBS-free DMEM cell culture medium. The tissue chunks were washed and treated with trypsin solution until the solution became turbid on standing. The trypsin solution was transferred into the FBS-containing DMEM cell culture medium and blended thoroughly. The cell suspension was then filtered using a gauze strainer (200 meshes) and centrifuged at $2500 \mathrm{r} / \mathrm{min}$ for $5 \mathrm{~min}$. The precipitate was scattered with FBS-containing DMEM and kept still for $2 \mathrm{~h}$. The cell suspension was then transferred into another bottle and mixed with 1\% bromodeoxyuridine (BRDU) in order to inhibit the growth of cardiac fibroblasts. Thereafter, the cells were incubated for $48 \mathrm{~h}$ and cultured for another $24 \mathrm{~h}$ before being used for the subsequent experimental procedures.

\section{Determination of cell viability}

Neonatal rat cardiomyocytes were seeded on 96-well plate and incubated with FBS-free culture medium containing 2\% SXSM serum and 2\% control serum in an incubator for $24 \mathrm{~h}$. Cells pretreated with FBS-free culture medium in the same volume served as control and model. Oxidative damage of cells was induced by replacing the FBS-free culture medium containing $150 \mu \mathrm{M} \mathrm{H}_{2} \mathrm{O}_{2}$. Cell viability was determined by CCK8 assay kit. In order to establish the anoxia-reoxygenation (A/R) injury model, neonatal rat cardiomyocytes were seeded on 96-well plate and incubated with FBS-free culture medium containing 2\% SXSM serum and $2 \%$ control serum in an incubator $\left(5 \% \mathrm{CO}_{2}\right)$ for $24 \mathrm{~h}$. Cells pretreated with FBS-free culture medium in the same volume served as a control. The cells were cultured under hypoxic conditions with $95 \% \mathrm{~N}_{2}$ and $5 \%$ $\mathrm{O}_{2}$ for $6 \mathrm{~h}$ followed by culturing under normoxic conditions for $12 \mathrm{~h}$. In the meantime, cells pretreated with FBS-free culture medium in the same volume under normoxic conditions served as control.

\section{Determination of redox parameters in cardiomyocytes}

Neonatal rat cardiomyocytes were seeded on 6-well plate and incubated with FBS-free culture medium containing 2\% SXSM serum and $2 \%$ control serum for $24 \mathrm{~h}$. The effects of SXSM serum on the redox parameters of cardiomyocytes including ROS, GSH, GSH-Px, and SOD were determined by respective assay kits.

Western blot

Anti-oxidative proteins levels were determined by western blot analysis. Neonatal rat cardiomyocytes were treated with ice-cold lysis buffer containing protease and phosphatase inhibitor. The lysate was centrifuged at $13,500 \mathrm{~g}$ at $4^{\circ} \mathrm{C}$ for $15 \mathrm{~min}$, and the supernatant was collected. Protein concentration was determined by bicinchoninic acid protein assay reagent kit. Samples were separated by electrophoresis on $12 \%$ sodium dodecyl sulfate polyacrylamide gel and transferred onto nitrocellulose membranes. Residual protein bands were blocked with 5\% milk and the membrane was incubated with rabbit anti-SOD1 antibody (1:1000) and rabbit anti-GCLC antibody (1:1000) dilution in phosphate buffered saline (PBS). Next, the membranes were incubated with HRP-conjugated, secondary anti-rabbit antibody at a 1:10000 dilutions in PBS. Protein levels were determined using the Odyssey Imaging System (LI-COR Biosciences, Lincoln, NE, USA). $\beta$-actin (1:4000) was used as an internal reference for data normalization.

\section{Data analysis}

Data were presented as means with standard deviations (SDs) and analyzed using $t$-test and one-way ANOVA analysis. $P$-value of less than 0.05 was regarded as statistically significant.

\section{Results}

SXSM protected myocardium from I/R injury

Hearts of SD rats treated with or without SXSM were subjected to myocardial I/R. Hearts treated without SXSM and perfused without I/R served as the control group. Hearts treated without SXSM and perfused with I/R served as the model group. Hearts treated without SXSM and perfused with IPC served as positive control. As illustrated in Fig. 1, heart rate, $\mathrm{LVDP},+\mathrm{dp} / \mathrm{dt}_{\max }$ and $-\mathrm{dp} / \mathrm{dt}_{\max }$ of the model group were significantly lower as compared to 
Fig. 1. SXSM protected cardiac function from myocardial I/R injury. (A) Perfusion procedures of each group. Control, the heart was perfused continuously without ischemia; $I / R$, the heart was perfused with $I / R ; I / R+I P C$, the heart was pretreated with IPC before I/R; I/R+SXSM, the heart was treated with SXSM before I/R. (B) Recovery of I/R injury-induced reduction in heart rate by SXSM and IPC. (C) Recovery of I/R injuryinduced reduction in LVDP by SXSM and IPC. (D) Recovery of I/R injury-induced reduction in $+\mathrm{dp} /$ $\mathrm{dt}_{\max }$ by SXSM and IPC. (E) Recovery of I/R injuryinduced reduction in $-\mathrm{dp} / \mathrm{dt}_{\max }$ by SXSM and IPC. ${ }^{*} \mathrm{P}<0.05,{ }^{* *} \mathrm{P}<0.01,{ }^{* * *} \mathrm{P}<0.001 ; \mathrm{n}=6$.

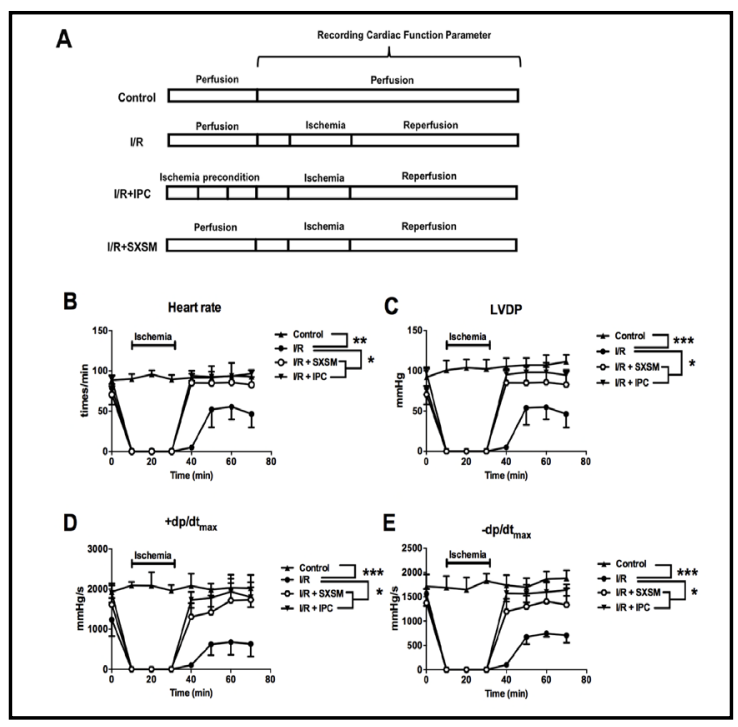

Fig. 2. Morphological changes and HE staining of heart tissue. (A) Heart before and after I/R injury. (B) HE staining of heart tissue after perfusion. Control, the heart was perfused continuously without ischemia; $I / R$, the heart was perfused with $I / R ; I / R+I P C$, the heart was pretreated with IPC before I/R; I/R+SXSM, administration of SXSM before I/R.

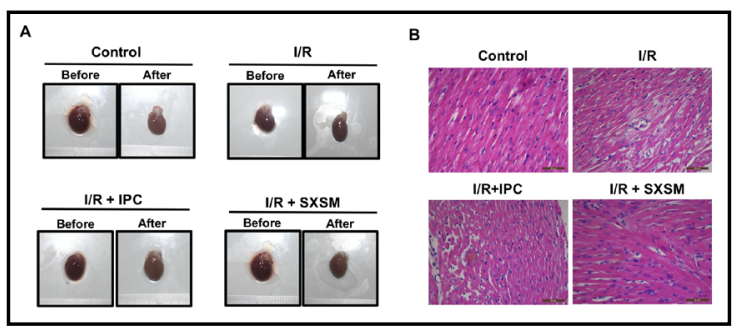

those of the control group after I/R $(P<0.01$ or $P<0.001)$. Treatment of SXSM and IPC significantly increased heart rate and improved LVDP, $+\mathrm{dp} / \mathrm{dt}_{\text {max }}$ and $-\mathrm{dp}_{\mathrm{d}} / \mathrm{dt}_{\text {max }}$ compared with model group $(P<0.05)$. The myocardial tissue of I/R group appeared to be more rigid and hard, and the heart lost its original shape. SXSM and IPC also effectively improved the above changes of heart induced by I/R injury. HE staining of the heart tissue showed that cardiomyocytes without I/R injury were well-arranged and distributed evenly and orderly. No abnormality was found in the structure and morphology of cardiomyocytes and myocardial interstitium. After I/R, the myocardial interstitium significantly expanded with an increase in cell size and granular degeneration of cardiomyocytes. After pre-treatment with SXSM or IPC, the expansion of myocardial interstitium, the structural abnormality and morphological changes of cardiomyocytes were corrected (Fig. 2). These results suggested that SXSM improved cardiac function, protected the structure and morphology of cardiomyocytes and the integrity of myocardium from I/R injury.

SXSM protected myocardium from I/R injury by increasing cardiac antioxidative capacity

In order to explore the mechanisms of SXSM responsible for cardiac function and structure protection from I/R injury, the antioxidant effects of SXSM on myocardium were further investigated. As illustrated in Fig. 3, SXSM significantly increased SOD activity, GSH-Px activity and GSH content in myocardium compared with model $(P<0.05$ or $P<0.01)$. Besides, SXSM significantly increased myocardial SOD1 and GCLC expression $(P<0.01)$. The results indicated that SXSM corrected cardiac dysfunction, protected the structure and morphology of cardiomyocytes and the integrity of myocardium from I/R injury by increasing cardiac antioxidant capacity. 
Fig. 3. SXSM prevented myocardial I/R injury by increasing myocardial antioxidant capacity. I/R, the heart perfused with I/R; I/R+SXSM, treatment of SXSM before I/R. (A) Increase in SOD activity by SXSM in myocardium with I/R injury. (B) Increase in GSH-Px activity by SXSM in myocardium with I/R injury. (C) Increase in GSH content by SXSM in myocardium with $I / R$ injury. (D) Increase in SOD1 expression by SXSM in myocardium with I/R injury. (E) Increase in GCLC expression by SXSM in myocardium with I/R injury. ${ }^{*} \mathrm{P}<0.05$, ${ }^{*} \mathrm{P}<0.01$ vs. $\mathrm{I} / \mathrm{R} ; \mathrm{n}=6$.

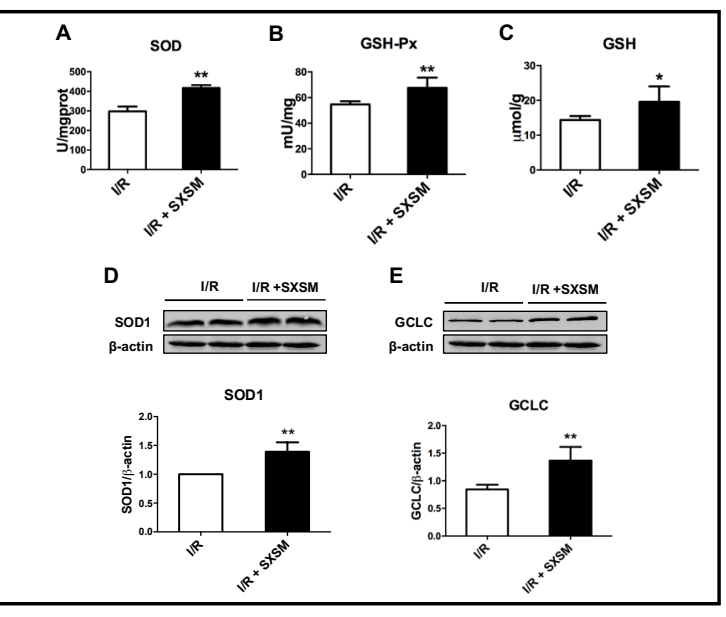

Fig. 4. HPLC analysis of SXSM serum, blank serum and SXSM. (A) HPLC analysis of blank serum. (B) HPLC analysis of the prepared SXSM serum. (C) HPLC analysis of SXSM.

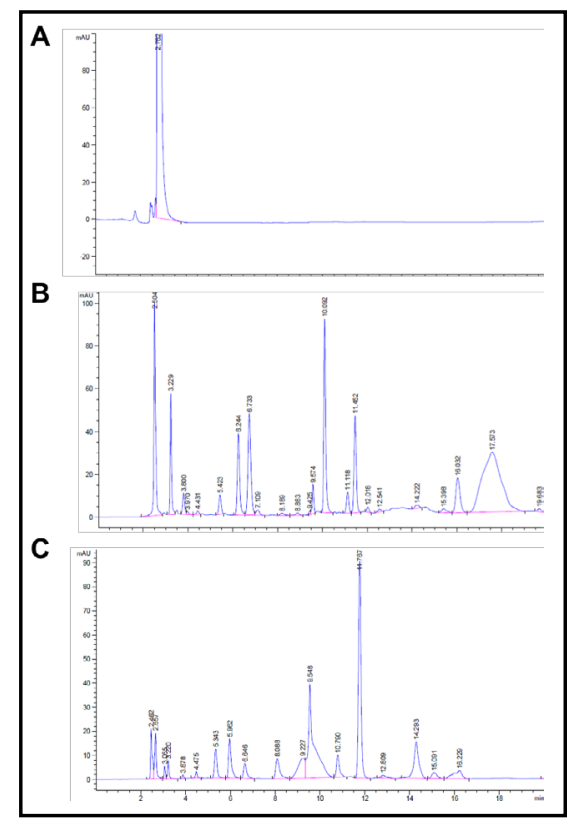

Fig. 5. SXSM protected cardiomyocytes from $\mathrm{H}_{2} \mathrm{O}_{2}$-induced oxidative damage by increasing antioxidant capacity. (A) Recovery of $\mathrm{H}_{2} \mathrm{O}_{2}$-induced reduction in cardiac cell viability by SXSM. (B \& C) Decrease in ROS level by SXSM in cardiomyocytes. (D) Increase in SOD activity by SXSM in cardiomyocytes. (E) Increase in GSH content by SXSM in cardiomyocytes. (F) Increase in GSH-Px activity by SXSM in cardiomyocytes. (G) Increase in SOD1 expression by SXSM in cardiomyocytes. (H) Increase in GCLC expression by SXSM in cardiomyocytes. ${ }^{*} \mathrm{P}<0.05,{ }^{* *} \mathrm{P}<0.01$ vs. Control; $\# \# \mathrm{P}<0.01$ vs. Model; $\mathrm{n}=6$.

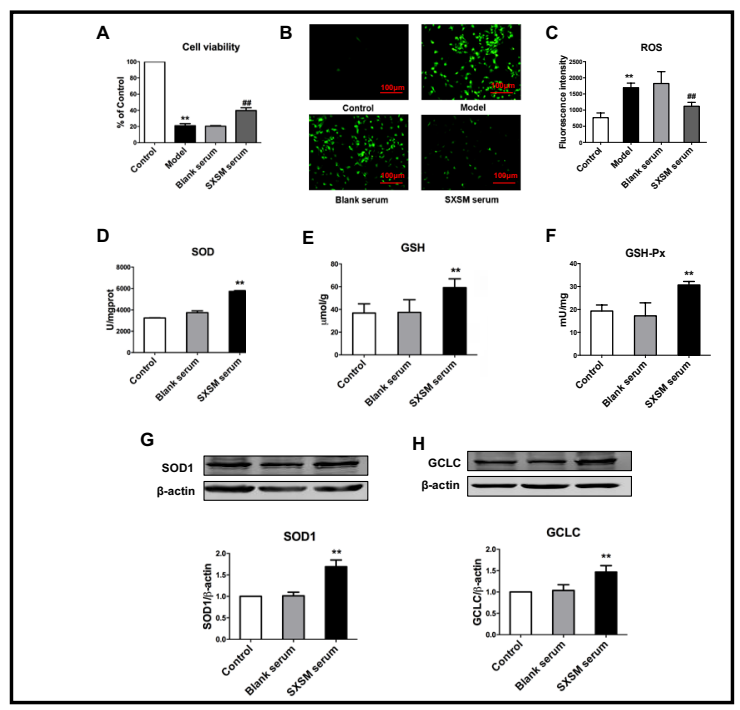


SXSM protected cardiomyocytes from antioxidative capacity

In order to investigate the reproducibility of the cardioprotective actions of SXSM against I/R injury at cellular level where cardiomyocytes are under oxidative stress, the main detrimental factor in I/R injury, we carried out the following steps. First, we prepared SXSM serum with serum pharmacology method and analyzed the differences of SXSM, control serum and SXSM serum using HPLC. It was confirmed that the prepared SXSM serum showed several significant absorption peaks which were the same as SXSM and different from the control serum, suggesting that SXSM serum was successfully prepared (Fig. 4). Second, we pretreated cardiomyocytes with $150 \mu \mathrm{M} \mathrm{H}_{2} \mathrm{O}_{2}$ to induce oxidative damage and changes of cell viability was determined after the pre-treatment. As shown in Fig. $5 \mathrm{~A}, \mathrm{H}_{2} \mathrm{O}_{2}$ led to significant reduction of cardiomyocyte viability, and notably, SXSM treatment ameliorated such reduction $(P<0.01)$. Moreover, SXSM decreased ROS in cardiomyocytes induced by Rosup reagent $(P<0.01$; Fig. 5B-C). SXSM also significantly increased activities of SOD, GSH-Px and GSH as well as expression of SOD1 and GCLC in cardiomyocytes $(P<0.01$; Fig. 5D-H).
$\mathrm{H}_{2} \mathrm{O}_{2}$-induced oxidative injury by increasing

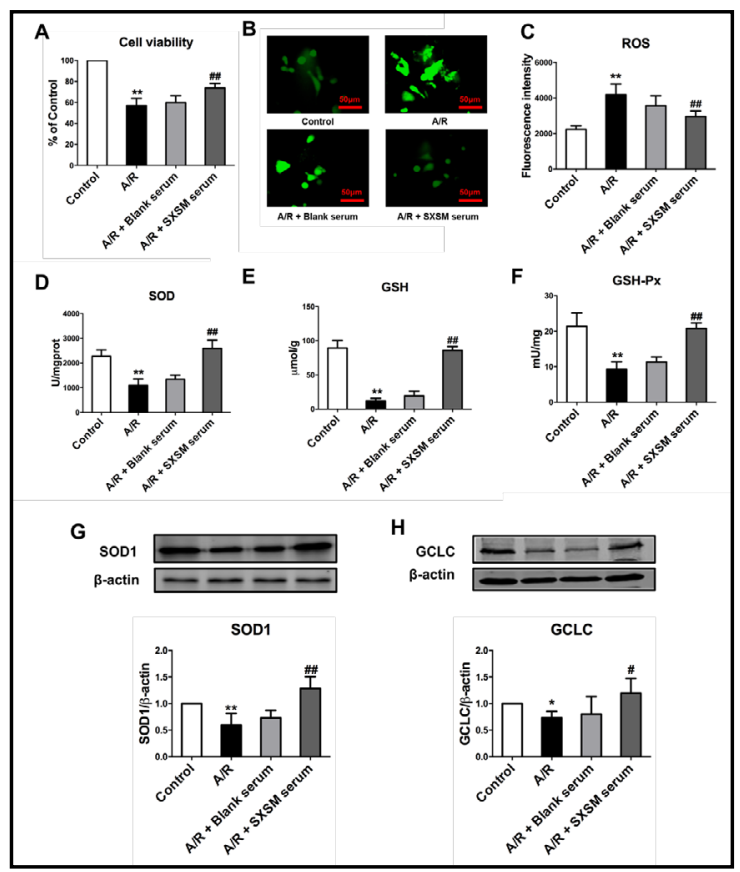

Fig. 6. SXSM protected cardiomyocytes from A/Rinduced oxidative damage by increasing antioxidant capacity. (A) Recovery of A/R injury-induced reduction in cardiac cell viability by SXSM. (B \& C) Mitigation of $\mathrm{A} / \mathrm{R}$ injury-induced overproduction of ROS by SXSM in cardiomyocytes. (D) Increase in SOD activity by SXSM in cardiomyocytes. (E) Increase in GSH content by SXSM in cardiomyocytes. (F) Increase in GSH-Px activity by SXSM in cardiomyocytes. (G) Increase in SOD1 expression by SXSM in cardiomyocytes. $(\mathrm{H})$ Increase in GCLC expression by SXSM in cardiomyocytes. ${ }^{*} \mathrm{P}<0.05$, ${ }^{* *} \mathrm{P}<0.01$ vs. Control; ${ }^{~} \mathrm{P}<0.05,{ }^{\# \#} \mathrm{P}<0.01$ vs. $\mathrm{A} / \mathrm{R} ; \mathrm{n}=6$.

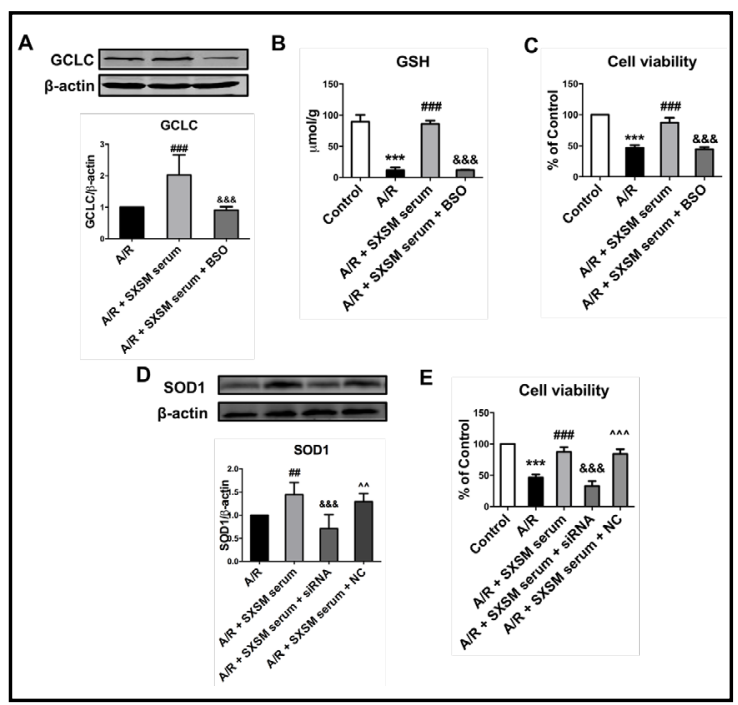

Fig. 7. BSO and SOD 1 siRNA counteracted the promoting effects of SXSM on GCLC and SOD1 expression, GSH content and cell viability. (A) Reversal of the SXSM-induced upregulation of GCLC expression by BSO in A/R injury cardiomyocytes. (B) Reversal of the SXSM-induced elevation of GSH content by BSO in A/R injury cardiomyocytes. (C) Reversal of the SXSM-induced increase in cardiomyocyte viability by BSO in A/R injury cardiomyocytes. (D) Reversal of the SXSM-induced upregulation of SOD1 expression by SOD1 siRNA in A/R injury cardiomyocytes. (E) Reversal of the SXSM-induced increase of cardiomyocyte viability by SOD1 siRNA in A/R injury cardiomyocytes. ${ }^{* * *} \mathrm{P}<0.001$ vs. Control; ${ }^{\# \#} \mathrm{P}<0.01$, \#\#\# $\mathrm{P}<0.001$ vs. $\mathrm{A} / \mathrm{R} ;{ }^{\& \&} \mathrm{P}<0.001$ vs. $\mathrm{A} / \mathrm{R}+\mathrm{SXSM}$ serum; ${ }^{\wedge} \mathrm{P}<0.01$, ${ }^{\wedge \wedge} \mathrm{P}<0.001$ vs. $\mathrm{A} / \mathrm{R}+\mathrm{SXSM}$ serum $+\mathrm{NC} ; \mathrm{n}=6$. 
Fig. 8. BSO counteracted the beneficial effects of SXSM on heart rate and cardiac function in myocardial I/R injury. (A) Reversal of the SXSMinduced elevation in heart rate by BSO in I/R injury myocardium. (B) Reversal of the SXSM-induced elevation of LVDP by BSO in I/R injury myocardium. (C) Reversal of the SXSM-induced elevation of $+\mathrm{dp} /$ $\mathrm{dt}_{\max }$ by BSO in I/R injury myocardium. (D) Reversal of the SXSM-induced elevation of $-\mathrm{dp} / \mathrm{dt}_{\max }$ increase by BSO in I/R injury myocardium. ${ }^{*} \mathrm{P}<0.05,{ }^{* *} \mathrm{P}<0.01$ vs. $\mathrm{I} / \mathrm{R}+\mathrm{SXSM} ; \mathrm{n}=6$.

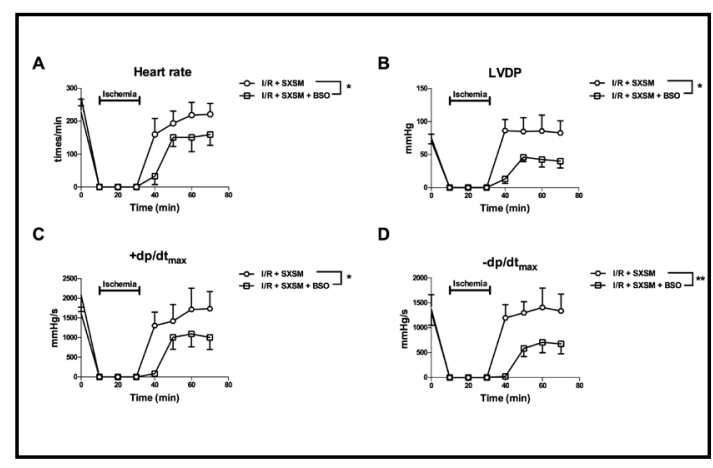

SXSM protected cardiomyocytes from A/R-induced oxidative injury by increasing antioxidative capactiy

Hypoxia is another key factor in ischemic injury and it can trigger overproduction of ROS. To further examine the antioxidative and cardioprotective effects of SXSM, cardiomyocytes were subjected to A/R injury with an anoxia workstation containing $95 \% \mathrm{~N}_{2}$ and $5 \% \mathrm{O}_{2}$ for $6 \mathrm{~h}$ followed by another $12 \mathrm{~h}$ culturing under normoxic conditions. Our results showed that A/R caused significant reduction of cardiomyocyte viability, and SXSM effectively reversed the deleterious change $(P<0.01$; Fig. 6A). In addition, SXSM decreased ROS content in cardiomyocytes induced by A/R injury $(P<0.01$; Fig. 6B-C). SXSM also significantly increased activities of SOD, GSH-Px and GSH as well as expressions of SOD1 and GCLC in cardiomyocytes with A/R injury $(P<0.01$; Fig. 6D-H $)$.

\section{Inhibition of GCLC and SOD1 counteracted the cardioprotective effects of SXSM}

To investigate whether GCLC and SOD1 could be targets of SXSM, the effects of L-buthioninesulfoximine (BSO), an inhibitor of GCLC, and SOD1 siRNA on the antioxidative and cardioprotective functions of SXSM were further assessed. Our results showed that BSO significantly inhibited SXSM-induced elevation in GCLC expression, GSH content and cell viabilities of A/R injury cardiomyocytes (Fig. 7A-C). The cardioprotective effects of SXSM were also abrogated by SOD1 siRNA in A/R injury cardiomyocytes, including SXSM-induced increases of SOD1 expression and cell viabilities of A/R injury cardiomyocytes (Fig. 7D-E). Moreover, after perfusion with BSO, heart rate was reduced and the improvement of cardiac function by SXSM was abrogated as reflected by a decrease in LVDP, $+\mathrm{dp} / \mathrm{dt}_{\text {max }}$ and $-\mathrm{dp} /$ $\mathrm{dt}_{\max }$ in the presence of SXSM and BSO (Fig. 8A-D). These results demonstrated that BSO and SOD1 significantly counteracted the protective effects of SXSM against oxidative damage. Therefore, SOD1 and GCLC may serve as potential therapeutic targets of SXSM in protecting the myocardium from I/R injury.

\section{Discussion}

Advances in research on the etiopathogenesis and management of ischemic heart disease have been made in recent years. However, ischemic heart disease is a very complex process affected by a wide spectrum of factors, which makes effective disease prevention and treatment difficult. The most effective therapy strategy is to shorten the duration of myocardial ischemia and to restore the blood perfusion as early as possible. However, recovery of blood flow further aggravates myocardial injury, leading to I/R injury and resulting in cardiac arrhythmias and myocardial dysfunction.

SXSM is a Chinese patent medicine used to treat bradyarrhythmias and sick sinus syndrome [18-23]. The present study provides novel experimental evidence on the protective effects of SXSM on myocardial I/R arrhythmias and cardiac dysfunction as well as insights on the mechanisms underlying the cardioprotective effects of SXSM. The current study found that SXSM reduced myocardial oxidative stress and protected cardiac function from 


\section{Cellular Physiology Cell Physiol Biochem 2018;48:2503-2516 \begin{tabular}{l|l|l} 
and Biochemistry & DOI: 10.1159/000492688 \\
Published 2018 The Author(s). Published by S. Karger AG, Basel \\
www.karger.com/cpb
\end{tabular}

I/R injury. Furthermore, SXSM protected cardiomyocytes from A/R injury by increasing ROS degradation through increasing SOD activity, GSH content and GSH-Px activity. Finally, SXSM increased SOD activity and GSH content by increasing expression of SOD1 and GCLC (Fig. 9). Therefore, SXSM may be a viable treatment option for myocardial I/R injury via its effects on SOD1 and GCLC.

Existing clinical studies showed that myocardial structure and function are often severely damaged during myocardial I/R injury. The most common clinical presentations of myocardial I/R injury are cardiac arrhythmias and myocardial stunning. A wide spectrum of factors, including intracellular $\mathrm{Ca}^{2+}$ overload, thrombin, platelet-activating factor, inositol triphosphate and angiotensin II, can adversely change the characteristics of transmembrane ion channels of cardiomyocytes during reperfusion, leading to $\mathrm{I} / \mathrm{R}$ arrhythmias. Myocardial I/R often causes ventricular premature beats and ventricular fibrillation, which lead to cardiac sudden death and become the main therapeutic targets of most anti-myocardial I/R drugs. However, many post-myocardial infarction patients suffer from long QT interval syndrome, characterized by the prolongation of action potential duration and bradyarrhythmias, which often lead to myocardial stunning and cardiac sudden death. Myocardial stunning refers to a continuous low-functioning state of the ischemic myocardium and it is observed in myocardial systolic and diastolic dysfunction induced by impaired ventricular function [25-27]. Our results demonstrated that SXSM significantly ameliorated bradyarrhythmias induced by I/R injury, indicating a possible anti-arrhythmic action of SXSM in myocardial I/R, which makes SXSM a unique pharmacological agent from other drugs with anti-I/R injury effects. In addition, SXSM improved cardiac systolic and diastolic function as indicated by increased LVDP, $+\mathrm{dp} / \mathrm{dt}_{\text {max }^{\prime}}$ and $-\mathrm{dp} / \mathrm{dt}_{\text {max }^{\prime}}$, which further illustrated the role of SXSM in alleviating myocardial I/R injury. Moreover, SXSM was shown to protect the structure and morphology of cardiomyocytes and the integrity of myocardium from I/R injury. Above all, our results confirmed that SXSM reduced myocardial I/R injury effectively by improving cardiac dysfunction and correcting the structural abnormalities and morphological changes of cardiomyocytes.

Though reperfusion is essential for the recovery of cardiac function, it induces further injury to myocardial tissues and cells. Reperfusion damages cardiomyocytes mainly through triggering the inflammatory response and inducing oxidative stress by producing ROS. Numerous studies have shown that the degree of myocardial cell death determines the severity of myocardial infarction. Therefore, viability of cardiomyocytes is a primary index when evaluating cardiac dysfunction during myocardial I/R injury [28, 29]. Existing research showed that A/R injury simulated myocardial I/R injury [30-31]. Therefore, in order to better study the effects of SXSM on I/R injury at the cellular level, an A/R injury cell model was established successfully in the present study. Our results demonstrated that A/R treatment significantly decreased the viability of cardiomyocytes and SXSM significantly inhibited the decrease of cell viabilities induced by A/R injury, suggesting that SXSM effectively protected cardiomyocytes from A/R injury. Our study findings provided further support to the theory of cardioprotective effects of SXSM on $\mathrm{H}_{2} \mathrm{O}_{2}$-induced oxidative damage, that is, SXSM significantly inhibited $\mathrm{H}_{2} \mathrm{O}_{2}$-induced cell apoptosis, indicating that SXSM protected cardiomyocytes from oxidative damage induced by oxygen free radicals. On the basis of above results, we speculated that SXSM protected cardiac function and cardiomyocytes from I/R injury through reducing oxidative stress and thus further research on the anti-oxidative effect of SXSM and the underlying mechanisms was pursued. 


\section{Cellular Physiology Cell Physiol Biochem 2018;48:2503-2516 \begin{tabular}{l|l} 
DOI: 10.1159/000492688 & $\begin{array}{l}\text { O 2018 The Author(s). Published by S. Karger AG, Basel } \\
\text { www.karger.com/cpb }\end{array}$
\end{tabular} \\ Zhao et al.: Shenxian-Shengmai Protects Myocardium from IR Injury}

ROS play a key role in myocardial I/R injury at the early stage of reperfusion. They are able to damage myocardium and cardiomyocytes during I/R [32, 33]. Our results showed that ROS level was significantly increased under A/R and SXSM suppressed such an elevation and protected the myocardium against oxidative stress. $\mathrm{O}_{2}{ }^{-}$is one of the major ROS. In living cells, SOD catalyzes $\mathrm{O}_{2}^{--}$to generate $\mathrm{H}_{2} \mathrm{O}_{2}$ and $\mathrm{O}_{2}$, which contribute to a reduction of ROS levels in cardiac cells. Therefore, SOD poses as the first-line target when defending against ROS and SOD is indeed considered to be the indispensable antioxidant in signal transduction and overall cellular function. $\mathrm{Cu} / \mathrm{ZnSOD}$ (SOD1) is the most abundant isoform of SOD in cytoplasm [34, 35]. Previous studies demonstrated that overexpression of SOD1 suppressed superoxide production, cell apoptosis and inflammation caused by I/R injury through inhibiting caspase-3 and caspase-9 [36]. Our results showed that SOD activity was decreased after myocardial I/R injury and cardiomyocyte A/R injury and SXSM significantly inhibited such detrimental effects. SXSM unregulated SOD1 expression in myocardial I/R injury and cardiomyocyte A/R injury, suggesting that SXSM increased myocardial SOD activity through the promotion of SOD1 expression. The above results collectively demonstrated that SXSM protected cardiomyocytes from oxidative damage during myocardial I/R injury and mitigated the subsequent development of cardiac dysfunction, probably due to an increase of SOD1 expression and SOD activity.

GSH-Px has peroxidase activity which protects the organism from oxidative damage. The biochemical functions of GSH-Px are to reduce toxic lipid hydroperoxides to their corresponding hydroxyl compouds and reduce free $\mathrm{H}_{2} \mathrm{O}_{2}$ to water, thus playing an important role after the conversion of $\mathrm{O}_{2}^{--}$to $\mathrm{H}_{2} \mathrm{O}_{2}$ catalyzed by SOD [37, 38]. GSH is the most abundant antioxidant in mammalian cells. It scavenges a variety of oxygen free radicals and protects cells from being damaged by ROS. Moreover, GSH is a major intrinsic cofactor for GSH-Px activity. Therefore, GSH is an important indicator when evaluating the body's antioxidant defense system $[39,40]$.GSH is synthesized by consecutive catalysis of $\gamma$-glutamylcysteinesynthetase and GCLC using cysteine, glutamate and glycine. BSO inhibits the activity of GCLC and thus inhibiting GSH synthesis [41, 42]. Extensive studies have confirmed that myocardial I/R injury reduced the levels of antioxidant enzymes such as GSH-Px and non-enzymatic antioxidants such as GSH, which inhibited their scavenging function and exacerbated cellular oxidative stress [43]. Our results showed that GSH content and GSH-Px activity were significantly deceased in I/R injury myocardium and A/R injury cardiomyocytes. However, SXSM was able to increase GSH content and GSH-Px activity effectively in both the I/R injury myocardium and $\mathrm{A} / \mathrm{R}$ injury cardiomyocytes, which contributed to a reduction in myocardial lipid peroxide and $\mathrm{H}_{2} \mathrm{O}_{2}$ levels, thus enhancing the body's antioxidative ability and reducing oxidative stress. Besides, SXSM significantly increased GCLC expression, the rate-limiting enzyme in GSH synthesis, in both I/R injury myocardium and A/R injury cardiomyocytes, suggesting that SXSM increased GSH content through promoting GCLC expression. However, BSO was shown to antagonize the effects of SXSM on GCLC expression, GSH content and cell viabilities. Moreover, BSO counteracted the protective effects of SXSM on cardiac function during myocardial reperfusion, confirming that GCLC served as a therapeutic target of SXSM in protecting the heart from oxidative damage and I/R injury. The above results, therefore, demonstrated that SXSM attenuated oxidative damage during myocardial I/R injury and prevented against the subsequent cardiac dysfunction probably due to increasing GSH-Px activity and GSH synthesis through promoting GCLC expression.

\section{Conclusion}

The present study investigated the pharmacological effects of SXSM in protecting the myocardium from I/R injury. One of our major findings is that SXSM elevated heart rate, improved cardiac dysfunction and corrected cardiac morphological and structural changes during myocardial $\mathrm{I} / \mathrm{R}$ and protected cardiomyocytes from oxidative stress induced by A/R injury and $\mathrm{H}_{2} \mathrm{O}_{2}$. Reduction in ROS might be one of the underlying mechanisms for the 


\section{Cellular Physiology Cell Physiol Biochem 2018;48:2503-2516 \begin{tabular}{l|l|l} 
and Biochemistry Published online: 16 August, 2018 & $\begin{array}{l}\text { @ } 2018 \text { The Author(s). Published by S. Karger AG, Basel } \\
\text { www.karger.com/cpb }\end{array}$ \\
\hline
\end{tabular}}

cardioprotective effects of SXSM. Increase in intracellular SOD activity, GSH content and GSHPx activity via upregulated expressions of SOD1 and GCLC might be the cellular mechanisms behind the antioxidative activity of SXSM. We therefore proposed that SXSM might be considered as an effective pharmacological agent for the treatment of myocardial I/R injury.

\section{Acknowledgements}

This work was conducted in the Department of Pharmacology of Harbin Medical University (State-Province Key Laboratories of Biomedicine-Pharmaceutics of China, Key Laboratory of Cardiovascular Medicine Research, Ministry of Education) and was financially supported by Application Technology and Development Project of Heilongjiang Province (grant number GA14C102), Central Guide Local Project of Science and Technology Development (grant number ZY16A07).

Yi-Xiu Zhao designed and performed the research study; Xin Zhang, Jing Luan and $\mathrm{Na}$ An performed the research study; Bu-Chang Zhao and Tao Zhao proposed the research study; Yan-Hong Sun and Zidong Donna Fu collected, analyzed and interpreted the data; Na Sun and Xin-Hui Wang performed the supplementary experiments; Yi-Xiu Zhao drafted the manuscript; Yan Zhang and Bao-Feng Yang revised the manuscript; all authors approved the final version of the manuscript.

\section{Disclosure Statement}

The authors declare that there are no conflict of interests.

\section{References}

-1 Ferdinandy P, Schulz R: Nitric oxide, superoxide, and peroxynitrite in myocardial ischemia-reperfusion injury and preconditioning. Brit J Pharmacol 2003;138:532-543.

- Hoffman JJ, Gilbert TB, Poston RS, Silldorff EP: Myocardial reperfusion injury: etiology, mechanisms, and therapies. J Extra Corpor Technol 2004;36:391-411.

3 Majidi M, Kosinski AS, Al-Khatib SM, Lemmert ME, Smolders L, van Weert A, Reiber JH, Tzivoni D, Bar FW, Wellens HJ, Gorgeis AP, Krucoff W: Reperfusion ventricular arrhythmia bursts predict larger infarct size despite TIMI 3 flow restoration with primary angioplasty for anterior ST-elevation myocardial infarction. Eur Heart J 2009;30:757-764.

4 Lopes RD, Li L, Granger CB, Wang TY, Foody JM, Funk M, Peterson ED, Alexander KP: Atrial fibrillation and acute myocardial infarction: antithrombotic therapy and outcomes. Am J Med 2012;125:897-905.

$>5$ He F, Zuo L: Redox Roles of reactive oxygen species in cardiovascular diseases. Int J Mol Sci 2015;16:2777027780.

-6 Zhu X, Zuo L: Characterization of oxygen radical formation mechanism at early cardiac ischemia. Cell Death Dis 2013;4:e787.

-7 Tollio F, Angotti C, Perrelli MG, Penna C, Pagliaro P: Redox balance and cardioprotection. Basic Res Cardiol 2013;108:392.

8 Perrelli M-G, Pagliaro P, Penna C: Ischemia/reperfusion injury and cardioprotective mechanisms: role of mitochondria and reactive oxygen species. World J Cardiol 2011;3:186-200.

-9 Penna C, Mancardi D, Rastaldo R, Pagliaro P: Cardioprotection: a radical view free radicals in pre and post conditioning. Biochim Biophys Acta 2009;1787:781-793.

10 Pagliaro P, Moro F, Tullio F, Perrelli MG, Penna C: Cardioprotective pathways during reperfusion: focus on redox signaling and other modalities of cell signaling. Antioxid Redox Signal 2011;14:833-850.

11 Rigoulet M, Yoboue ED, Devin A: Mitochondrial ROS generation and its regulation: mechanisms involved in $\mathrm{H}_{2} \mathrm{O}_{2}$ signaling. Antioxid Redox Signal 2011;14:459-468. 


\section{Cellular Physiology Cell Physiol Biochem 2018;48:2503-2516 \begin{tabular}{l|l|l} 
and Biochemistry Published online: 16 August, 2018 & $\begin{array}{l}\text { C) 2018 The Author(s). Published by S. Karger AG, Basel } \\
\text { www.karger.com/cpb }\end{array}$ \\
\hline
\end{tabular}}

12 Jiang YQ, Chang G, Wang Y, Zhang DY, Cao L, Liu J: Geniposide prevents hypoxia/reoxygenation-induced apoptosis in $\mathrm{H}_{9} \mathrm{C}_{2}$ cells: improvement of mitochondrial dysfunction and activation of GLP-1R and the $\mathrm{PI}_{3} \mathrm{~K} /$ AKT signaling pathway. Cell Physiol Biochem 2016;39:407-421.

13 Schriewer JM, Peek CB, Bass J, Schumacker PT: ROS-mediated RAPP activity underlines mitochondrial function after permeability transition pore opening during myocardial ischemia-reperfusion. J Am Heart Assoc 2013;12:e000159.

-14 Cha MJ, Jang JK, Ham Q Song BW, Lee SY, Park JH, Lee J, Seo HH, Choi E, Jeon WM, Hwang HJ, Shin HT, Choi E, Hwang KC: MicroRNA-145 suppresses ROS-induced $\mathrm{Ca}^{2+}$ overload of cardiomyocytes by targeting CaMKIIס. Biochem Biophys Res Commun 2013;435:720-726.

-15 Vaage, J, Antonelli M, Bufi M, Irtun O, DeBlasi RA, Corbucci GG, Gasparetto A, Semb AG: Exogenous reactive oxygen species deplete the isolated rat heart of antioxidants. Free Radic Biol Med 1997;22:85-92.

16 Jain AK, Mehra NK, Swarnakar NK: Role of antioxidants for the treatment of cardiovascular diseases: challenges and opportunities. Curr Pharm Des 2015;21:4441-4455.

17 Chen HJ, Zhang H, Gu NF, Pan J, Sun RY, Zhao C, Du QM: Analysis of curative effect of Shenxian-shengmai oral liquid in the treatment of 56 cases of bradyarrhythmias. Chin J Ethn 2013;4:99.

$18 \mathrm{Hu}$ JH, Chen SJ, Hua XL: Clinical research of Shenxianshengmai oral liquid in the treatment of sick sinus syndrome and sinus bradycardia. Chin Tradition Patent Med 2012;34:7-9.

19 Li P, Zeng L: Clinical observation of Shenxian-shengmai oral liquid in the treatment of bradycardia. Chin J Integr Med Cardio 2011;9:1121-1122.

20 Hou P, Zhu F, Ding MY, Liu N, Ding KF: Application of ultrasonic to evaluate the effect of Chinese herbal compound on the cardiac function of bradyarrhythmic rats. Arch Tradit Chin Med 2013;31:1732-1733.

21 Liu ZY, Huang J, Liu NN, Zheng M, Zhao T, Zhao BC, Wang YM, Pu JL: Molecular mechanisms of increased heart rate in shenxianshengmai-treated bradycardia rabbits. Chin Med J (Engl) 2017;130:179-186.

22 Tang H, Li J, Huang ZH: Clinical observation on treatment of sick sinus syndrome with Shenxianshengmai oral liquid. Chin Med 2010;5:979-980.

23 Xi SF, Cheng W, Shi XJ, Long ZX: Effect of Shengxian-shengmai liquid on the sick sinus model rabbits through duodenal administration. J Beijing Univ Trad Chin Med 1999;22:34-36.

24 Xi SF, Wu SR, Yan XJ, Guo Y, Shi XJ, Long ZX, Liu AL: Pharmacodynamical effects of Shenxianshengmai liquid on activity blood stasis. Tradit Chin Drug Res Pharmacol 1999;10:211-212.

25 Temsah RM, Netticadan T, Dhalla NS: Mechanisms of cellular alterations due to ischemia-reperfusion injury in the heart. Myocardial ischemia and preconditioning; in: Dhalla NS, Takeda N, Singh M, Lukas A (eds): Myocardial Ischemia and Preconditioning. Progress in Experimental Cardiology. Boston, Springer, 2003, vol 6, pp 149-164.

-26 Lerner DL, Yamada KA, Schuessler RB, Saffitz JE: Accelerated onset and increased incidence of ventricular arrhythmias induced by ischemia in Cx43-deficient mice. Circulation 2000;101:547-552.

27 Lim KH, Lim DJ, Kim JH: Ginsenoside-re ameliorates ischemia and reperfusion injury in the heart: A hemodynamics approach. J Ginseng Res 2013;37:283-292.

-28 Eefting F, Rensing B, Wigman J, Pannekoek WJ, Liu MM, Cramer MJ, Lips DJ, Doevendans P: Role of apoptosis in reperfusion injury. Cardiovasc Res 2004;61:414-426.

-29 Holleyman CR, Larson DF: Apoptosis in the ischemic reperfused myocardium. Perfusion 2001;16:491-502.

-30 Xu J, Hu H, Chen B, Yue R, Zhou Z, Liu Y, Zhang S, Xu L, Wang H, Yu Z: Lycopene protects against hypoxia/ reoxygenation injury by alleviating ER stress induced apoptosis in neonatal mouse cardiomyocytes. PLoS One 2015;10:e0136443.

-31 Li X, Zeng Z, Li Q, Xu Q, Xie J, Hao H, Luo G, Liao W, Bin J, Huang X, Liao Y: Inhibition of microRNA-497 ameliorates anoxia/reoxygenation injury in cardiomyocytes by suppressing cell apoptosis and enhancing autophagy. Oncotarget 2015;6:18829-18844.

-32 Rodrigo R, Libuy M, Feliu F, Hasson D: Oxidative stress-related biomarkers in essential hypertension and ischemia-reperfusion myocardial damage. Dis Markers 2013;35:773-790.

-33 Braunersreuther V, Jaquet V: Reactive oxygen species in myocardial reperfusion injury: from physiopathology to therapeutic approaches. Curr Pharm Biotechnol 2012;13:97-114.

34 Zhou TY, Chuang C, Zuo L: Molecular characterization of reactive oxygen species in myocardial ischemiareperfusion injury. Biomed Res Int 2015;2015:864946. 


\section{Cellular Physiology Cell Physiol Biochem 2018;48:2503-2516 \begin{tabular}{l|l|l|} 
DOI: 10.1159/000492688 & $\begin{array}{l}\text { O 2018 The Author(s). Published by S. Karger AG, Basel } \\
\text { www.karger.com/cpb }\end{array}$ \\
\hline
\end{tabular}

35 Schneider MP, Sulivan JC, Wach PF, Boesen EI, Yamamoto T, Fukai T, Harrison DG, Pollock DM, Pollock JS: Protective role of extracellular superoxide dismutase in renal ischemia/reperfusion injury. Kidney Int 2010;78:374-381.

36 Tanaka MD, Mokhtari GK, Terry RD, Balsam LB, Lee KH, Kofidis T, Tsao PS, Robbins RC: Overexpression of human Copper/Zinc superoxide dismutase (SOD1) suppresses ischemia-reperfusion injury and subsequent development of graft coronary artery disease in murine cardiac grafts. Circulation 2004;110:II200-206.

-37 Wu G, Fang Y-Z, Yang S, Lupton JR, Turner ND: Glutathione metabolism and its implication for health. J Nutr 2004;134:489-492.

38 Robertson RP, Harmon JS: Pancreatic islet $\beta$-cell and oxidative stress: The importance of glutathione peroxidase. FEBS Letters 2007;581:3743-3748.

39 Lu SC: Regulation of glutathione synthesis. Mol Aspects Med 2009;30:42-59.

-40 Yoshioka Y, Kadoi H, Yamamuro A, Ishimaru Y, Maeda S: Noradrenaline increases intracellular glutathione in human astrocytoma U-251 MG cells by inducing glutamate-cysteine ligase protein via p-38 adrenoceptor stimulation. Eur J Pharmacol 2016;772:51-61.

41 Meister A: Glutathione Metabolism. Methods Enzyhmol 1995;251:3-7.

42 Zhang Y, Sano M, Shinmura K, Tamaki K, Katsumata Y, Matsuhashi T, Morizane S, Ito H, Hishiki T, Endo J, Zhou H, Yuasa S, Kaneda R, Suematsu M, Fukada K: 4-Hydroxy-2-nonenal protects against cardiac ischemiareperfusion via the Nrf2-dependent pathway. J Mol Cell Cardiol 2010;49:576-586.

$\$ 43$ Braunersreuther V, Jaquet V: Reactive oxygen species in myocardial reperfusion injury: from physiopathology to therapeutic approaches. Curr Pharm Biotechnol 2012;13:97-114. 